\title{
Ultrafast electron microscopy of electromagnetic waveforms
}

\author{
Andrey Ryabov ${ }^{1,2}$ and Peter Baum ${ }^{1,2,3,}{ }^{*}$ \\ ${ }^{1}$ Ludwig-Maximilians-Universität München, Am Coulombwall 1, 85748, Garching, Germany \\ ${ }^{2}$ Max-Planck-Institute of Quantum Optics, Hans-Kopfermann-Straße 1, 85748, Garching, Germany \\ ${ }^{3}$ Universität Konstanz, Universitätsstraße 10, 78464 Konstanz, Germany
}

\begin{abstract}
We advance ultrafast electron microscopy from atomic motions into the domain of electron-dynamics. Sub-light-cycle electron pulses deflected by time-frozen fields reveal the electromagnetic fields around a metamaterial element with sub-cycle and sub-wavelength resolution.
\end{abstract}

\section{Introduction}

Electron wavelength at several hundred of kiloelectronvolt is $100,000 \times$ smaller than wavelength of light and therefore it allows resolving the atoms directly in space. Ultrafast pump-probe imaging has been used to measure many kinds of structural dynamics, but not yet purely electromagnetic phenomena, although rapidly oscillating charges are the basis of optics and also define properties and operation of almost any information processing device or metamaterial.

\section{Sub-cycle imaging in space and time}

Here we report waveform electron microscopy, which enhances a transmission electron microscope with a sub-cycle and subwavelength access to electromagnetic phenomena [1]. Femtosecond electron pulses (blue, Fig. 1) are photoemitted by a pulsed laser and compressed in time by single-cycle $\mathrm{THz}$ filed (red). The beam passes through the sample, for example, a split-ring resonator (yellow) that is excited with a second single-cycle pulse (red). The time-compressed electron pulses [2] are $\sim 15$ times shorter than the excitation half-cycle. Therefore, the fields that locally

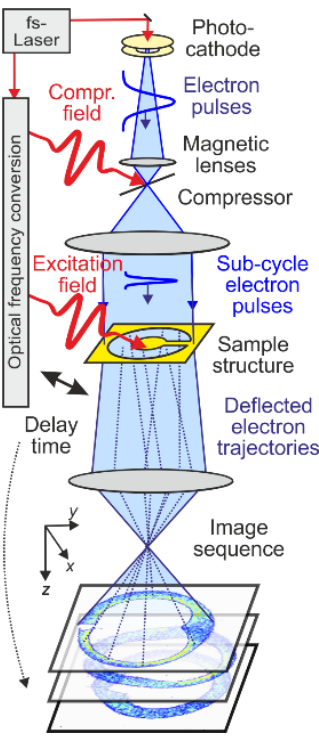

Fig. 1. Concept and experimental setup (TEM geometry). Optical pulse compresses electrons in time and also triggers electromagnetic resonances in the sample.

\footnotetext{
${ }^{*}$ Corresponding author: Peter.baum@uni-konstanz.de
} 
deflect electrons in the sample are frozen in time for electrons, and these deflections are proportional to the instantaneous electromagnetic field vectors via the quasi-classical Lorentz force. A pump-probe sequence of deflected electron images contains information of the electromagnetic field vectors in the sample.

\section{Transmission imaging}

In transmission electron microscope (TEM) geometry [1], a well-collimated electron beam illuminates a sample and distorted electron shadow images are acquired as it is shown in Fig. 1. Figure 3(b) shows some results in form of three vector-field snapshots at different delay times. The field at $2.8 \mathrm{ps}$ reveals three local maxima, an asymmetry in $\mathrm{x}$, a predominantly radial polarization, and field enhancement at metal edges. The field first is localized at the gap and top half according to excitation field polarization, and after evolves in a longer-lived mode with mostly radial polarization. Time traces of the polarization can be evaluated at every point providing maps of central frequencies, dampings and group delays, which can be interpreted in collective charge density dynamics (see Fig. 3(c) and Fig. 3(d), respectively).

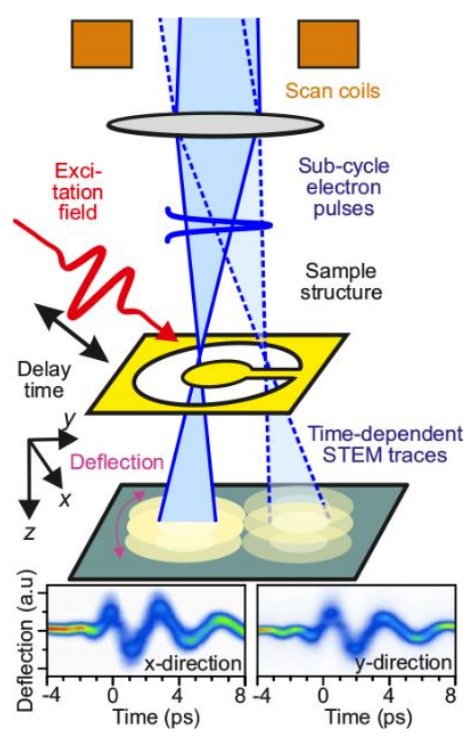

Fig. 2. Scetch setup of subcycle scanning transmission electron microscopy. Preliminary results are shown in lower panels.

\section{Scanning imaging}

A step forward is sub-cycle imaging in a scanning-TEM (STEM) regime (see Fig. 2). The deflection amplitude of the focused beam and its width provide information about local fields in the sample. Waveform STEM instead of waveform TEM can provide better spatial resolution, surface and topographical sensitivity, while at the same time reducing the

(a)

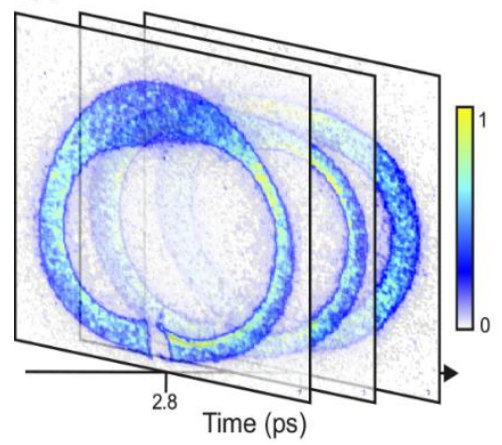

(b)

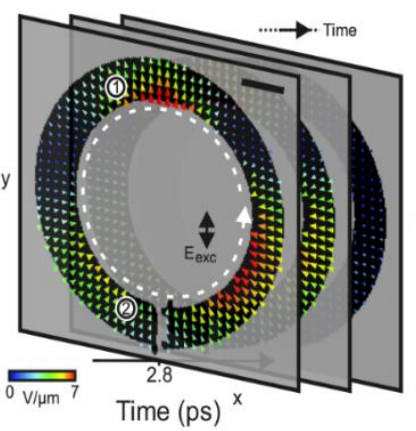

(c)

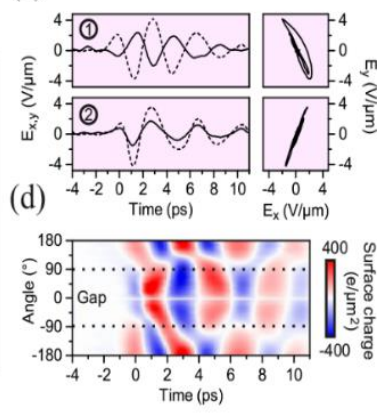

Fig. 3. Transmission electron microscopy of electromagnetic waveforms. (a) Subset of raw shadow images. (b) Time-dependent fields. (c) Polarizations at locations (1) and (2) in (b). (d) Space-time map of the surface carrier density at the inner edge; dashed line in (b). 
computation power needed for analysis. The resolution of STEM waveform imaging, however, is limited by focusing angle of the beam. The lower panels in Fig. 2 show preliminary results of sub-cycle deflection recorded at one point of a split-ring resonator.

\section{Conclusion and remarks}

Sub-cycle electron dynamics on atomic dimensions is the origin of all linear or nonlinear optical effects while collective carrier dynamics determines the performance of ultrafast electronic and nanophotonic devices. Our waveform electron microscopy, in TEM or STEM geometry, should contribute to revealing such dynamics in space and time. Magnetic fields, which are the essence of negative-index metamaterials or memory devices, can be revealed and separated from the electric-field deflections by making two experiments at different electron velocities. Techniques, which are used in this concept, can also reveal static electric or magnetic fields with high resolution. Energy-filtered imaging can measure forward momentum changes and in combination with tomography reveal the complete electric and magnetic vector fields in space and time. Merging electron waveform microscopy with attosecond science, as recently reported in a proof-of-concept $[3,4]$, will be an extremely powerful instrument to study electron motions driven by visible light. Instrumental advances and novel ultrafast capabilities of modern TEMs [5] will directly apply to our concept.

\section{References}

1. A. Ryabov and P. Baum, Science 353, 374 (2016)

2. C. Kealhofer et al., Science 352, 429 (2016)

3. Y. Morimoto and P. Baum, Nature Phys. 14, 252 (2018)

4. Y. Morimoto and P. Baum, Phys. Rev. A 97, 033815 (2018)

5. K. E. Priebe et al., Nature Phot. 11, 793 (2017) 